\title{
ESTUDIOS
}

\section{[Movilidad de la población para enfrentar los cambios en el sector rural]}

\section{Ramón Valderas Ojeda}

Facultad de Ciencias Agrarias y Forestales, Universidad de Chile

\section{RESUMEN}

La realidad del mundo rural en el presente no se mide por la proporción de la población que vive en él, ni por su aporte a la economía del país. Ahora adquiere importancia fundamental su papel en la integridad del territorio, la conservación de los recursos naturales y la capacidad para proporcionarle a toda la población un disfrute de vida que es cada vez más apreciado. Esta nueva dimensión emerge, en buena parte, por los niveles críticos que alcanza la contaminación ambiental, los traslados agotadores de la residencia al trabajo, los riesgos y otros graves problemas del sistema urbano.

En este trabajo se resumen las nuevas condiciones que están apareciendo en el sistema de vida rural y en la misión destino de esta parte de la nación. Estas condiciones están dadas, en primer lugar, por la mayor comprensión teórica por parte del gobierno, las instituciones públicas y organizaciones privadas del territorio, en un país semivacío, como el nuestro, y donde se ha llegado a un sistema de vida en que se anhelan el paisaje, la tranquilidad y el espacio. Además, los propios habitantes rurales adoptan estrategias que se mencionan en el artículo, las que vienen a constituir respuestas no programadas al desafío de supervivencia. Asimismo, otra condicionate contribuye a explicar la posibilidad de cambios; esta es que los servicios a las industrias se están acercando a los sectores rurales, tanto por razones de avance tecnológico como por razones económicas de localización. Se repasan, entonces, las consecuencias que tienen los cambios previstos en la movilidad de la población y en particular en la migración interna y en las combinaciones de trabajos urbano-rurales que emergen a la luz de algunos instrumentos de análisis, provenientes de la geografía y la economía regional.

\section{Introducción}

El empleo, en la agricultura y otras actividades primarias, ha descendido notablemente en su participación dentro de la fuerza de trabajo nacional. Al mismo tiempo el empleo en los servicios, en especial en el área financiera, en las relaciones comerciales de exportación, en comunicaciones, en informática y en previsión y salud privadas, ha crecido permanentemente. La ocupación en la industria sigue siendo importante, pero no ha experimentado crecimientos porcentuales espectaculares. Por otro lado la población se ha urbanizado 
crecientemente dentro de un patrón de "concentración dispersa", permitida por el gran aumento de automóviles, la extensión del Metro y en general de los medios de transporte y de las vías. Paralelamente es posible observar cómo algunas comunas se despueblan lentamente, mientras crecen los centros urbanos clásicos de la Región Metropolitana, la $\vee$ y la VIII regiones. La combinación de situaciones hacen que la urbanización y el desarrollo regional sean problemas gemelos. Se impone un examen profundo de los nuevos procesos que advienen mediante las nuevas tecnologías de comunicación, transporte e informática, cuyas consecuencias, también operan en el antiguo y clásico sector "rural".

Asimismo, la preocupación de muchos expertos y del público por la temática conservacionista, el equilibrio ecológico y la equidad en el disfrute del territorio y de los ingresos, cobra cada vez con mayor importancia en los sectores dirigentes, por lo que podría preverse algún resultado práctico sobre la gestión del territorio.

En efecto, conviene abordar la prognosis de cuál y de qué magnitud serán las propensiones a vivir en pueblos medianos, pequeños o en condominios rurales cerca de la fuente de trabajo, sin estar aislados de la gran ciudad y acompañados de la posibilidad de disfrutar de muchas de las antiguas ventajas que ahora ya no son exclusivas de ésta. Si el fenómeno tiene relevancia para las decisiones de las familias y las personas, modulará en alguna proporción el traslado de la gente a lugares que garanticen una calidad de vida adecuada o que procuren ventajas no obtenibles en el centro de las metrópolis o en sus barrios. Este es el fondo de la materia que se trata en este estudio, afirmándose en el conocimiento de algunas experiencias directas o asociadas a estudios realizados en la Universidad de Chile.

\section{Movilidad de las personas y la urbe}

La movilidad de las personas, en su acepción tradicional, se refiere preferentemente al cambio de residencia dentro de áreas sujetas a la influencia urbana. Un referente importante es la clásica obra de Dickinson (1), en la que, aludiendo a las ciudades británicas, señala que en las grandes ciudades industriales hay una disminución de la población en el centro de la ciudad, donde edificios comerciales reemplazan a las casas vacías y hay un tremendo incremento en las franjas que la rodean, y donde el campo se reemplaza por suburbios y pueblos. Ya en 1921 en Gran Bretaña, se experimentaba el fenómeno llamado commuting que detentan los habitantes de áreas residenciales vinculadas a las conurbaciones donde se desarrolla el trabajo diario. Estas áreas residenciales, para el caso de Inglaterra, estaban separadas del trabajo por una o dos horas en tiempo. Así entonces, la movilidad ha estado corrientemente asociada a la gran ciudad ya sea asimilada a la extensión de ella o a su reestructuración interna.

En Chile esos fenómenos se han experimentado en los últimos años con una 
particular intensidad en la redistribución de la población hacia la periferia con abandono de los lugares centrales. Los Censos de población revelan cómo las comunas de Santiago, Providencia, Quinta Normal y Recoleta, que son constituyentes del centro, han disminuido su población.

El inducir la extensión de la ciudad hacia el campo no es necesariamente una invasión negativa de la gran ciudad, puesto que pueden concatenarse con la vida en el campo sin erosionar ni ocupar zonas críticas agrícolas que en el caso de Santiago, es posible que ya estén prácticamente agotadas. Desde hace tiempo en la Región Metropolitana se han estado invadiendo cerros, colinas o terrenos no agrícolas. En la zona de Concepción, donde no hay mucha tierra agrícola, la expansión se da en los antiguos arenales formados por el Bío-Bío, o en las diversas quebradas y colinas hasta Tomé, o bien en zonas de relleno hacia Talcahuano y en toda una zona donde predominan los suelos de aptitud forestal. En el caso de Temuco, Punta Arenas, Antofagasta, Iquique, Arica, Coquimbo, Puerto Montt, Ancud, Castro, Aisén la existencia de tierra agrícola de buena calidad es muy limitada, aunque hay importantes extensiones de tierra de pastoreo disponibles; es difícil imaginarse la invasión de territorio propiamente agrícola. En todo caso es importante saber que está sucediendo en Rancagua, Buin, San Fernando, Curicó, Talca, Linares, Parral, Los Angeles, Angol, y otras ciudades del Valle Central.

El fenómeno de expansión de la población urbana sobre áreas rurales se ha visto reflejada dramáticamente por las comunas de la Florida (2) y Maipú (3), comunas de tamaño similar; y por los últimos crecimientos explosivos de población que se han experimentado en Puente Alto (4) y, también en La Pintana (5), ambas comunas localizadas en la zona Sur de Santiago.

\section{Movilidad y migración rural}

El tema de la movilidad está también vinculado a la migración campo-ciudad que ha sido particularmente intensa en los últimos cincuenta años. Esto añade un movimiento poblacional con efectos de largo alcance, concerniente a la forma de ocupar los espacios urbanos de las grandes ciudades. También simultáneamente con aumentar la población urbana, ocurre que los habitantes rurales crecen a tasas menores que el promedio nacional; $y$, que en algunos casos aparece un estancamiento y despoblamiento de sectores rurales lo que normalmente va acompañado de marginalidad social y pobreza.

Preocupados por este fenómeno de la localización urbano rural de la población de Chile, Valderas y colaboradores (6) plantearon un modelo de crecimiento nacional con maximización del empleo o del ingreso, considerando una distribución espacial que hiciera crecer levemente el porcentaje de la población rural y su entorno urbano en provincias y regiones. El modelo obligaba, en el fondo, a hacer crecer unos cincuenta pueblos comunas para recibir en ellos el crecimiento vegetativo que iba a las capitales regionales y a los grandes centros 
metropolitanos del país, y al excedente de población rural que continuaría ejerciendo actividades ligadas a la producción primaria o a otras producciones $\mathrm{y}$, también a servicios ligados al entorno rural.

Posteriormente, y una vez que se dispuso de los datos del Censo de Población de 1992, se hizo un estudio de la evolución del crecimiento de la población en las comunas agrupadas según su grado de ruralidad, industrialización, densidad, superficie y población inicial, según estimaciones de la población de las nuevas comunas, corregidas por el Departamento de geografía del INE y cuyos resultados están en una publicación del Instituto de Estudios Regionales de la Universidad de Chile (7).

La tendencia de las comunas es relativamente interesante y está reflejando algunos cambios que es importante explorar. El primero de ellos es que se constata que, en una cantidad de 57 de las 335 comunas al año 1992, se experimentó una disminución efectiva de la población, entre el Censo de 1982 y el de 1992. Estas comunas están concentradas en 6 áreas relativamente identificadas: comunas del altiplano; comunas del secano costero de la VI, la VII y la VIII regiones; comunas áridas de la IV región; comunas de la precordillera de la VIII y IX región; la zona costera de la IX región, donde hay una importante población de origen mapuche; y finalmente, algunas comunas de Chiloé.

Las comunas deprimidas, o sea las 57 ya mencionadas, disminuyen en un $20 \%$ su población en términos absolutos. Por otra parte, el resto de las comunas de contenido rural o agrícola-forestal han aumentado su población en un equivalente al $17 \%$ de los habitantes originales. Sin embargo, el crecimiento experimentado por estas comunas es insuficiente para alcanzar la meta propuesta por el modelo citado, al año 2000. Como puede apreciarse en este estudio no se están aplicando conceptos de rural y urbano de manera clásica. Esto se debe a que el modo de vida de los pueblos y de algunas ciudades de regiones se complementan y dependen de la actividad rural, ya sea pesquera, forestal, agrícola, minera, ganadera o de otro tipo. Por lo tanto, se actúa con la premisa que considerar el mundo rural ajeno a los centros de servicios es un equivocación. En este entendido se han definido las agrupaciones de comunas con dependencia y alto contenido rural. Estas constituían en 1982 un $28.65 \%$ de la población del país y las mismas en 1992, habían descendido a un $27.41 \%$ de la población total. Si la tendencia continuara igual a la del período 1982-1992, la proporción llegaría a un $26.44 \%$ de la población del país al año 2000 . Algunas de las razones para pensar en una relativa estabilidad de la poblacional regional, estaría en la consolidación de villorrios en municipios rurales; la generación de actividades proveniente de la explotación de recursos naturales y su industrialización; el crecimiento de las exportaciones de productos forestales y del agro, de la pesca y del cultivo de especies marinas y lacustres; la mejora e implantación de servicios locales y comunales; y, la mejora de ciertas infraestructuras (8). 
Otro hecho relevante es que existe no sólo una inercia para cambiar de residencia, sino que, si se dan las condiciones, las familias se adaptan de tal forma que pueden asumir nuevas ocupaciones y reacomodar su forma de vida las nuevas circunstancias.

La migración neta de las diferentes regiones ha sido negativa en todas las regiones históricamente, salvo en la I, la Región Metropolitana y la XII Región y las proyecciones de migración neta del INE van desde -10 personas por mil al año hasta -0.1 persona por mil al año (9). Se constata por mediciones realizadas en Lolol, Romeral y Teno (localidad de Comalle) que la tasa neta experimentada por Romeral y Teno, comunas agrícolas de la provincia de Curicó presenta una tendencia más variada. El resultado es positivo para Romeral y levemente negativo para Comalle. En esta, última localidad de Teno se producen dos fenómenos contrapuestos: una alta inmigración y una alta emigración. Este caso se explica porque el sector está sujeto al crecimiento e influencia urbana de Curicó, y por su intermedio a otras ciudades y al crecimiento del sector frutíhortícola en el área de riego.

¿Dónde se comporta la migración como señalaba el INE?: en Lolol.

Lolol es una comuna del secano costero, alejada de ciudades importantes, excepto de Santa Cruz, que a su vez presenta un estancamiento poblacional. En ella la inmigración es muy pequeña porque en la actualidad no existe sino una ganadería y agricultura de secano con una pequeña área regada con muy limitada expectativa de empleo. Sin embargo, Lolol ocupa una parte importante del valle del Nilahue donde existen tierras de aptitud agrícola y, sólo, porque no se ha implementado el desarrollo completo del proyecto de riego de Convento Viejo, no están en producción 40.000 hectáreas de primera calidad (10). Por otro lado el destino de la emigración rural que era preferentemente a la ciudad de Santiago hasta el quinquenio 1975-1980, en los períodos 1980-1985 y 19851990 cambió de dirección para volcarse a la propia región de origen en otras comunas con agricultura de riego. Al profundizar un poco más acerca de este fenómeno se comprobó además, corroborando estudios realizados en la Universidad de Chile y otros, que los temporeros y pensionados de las comunas de Teno y Lolol ascendían a porcentajes importantes en relación a la fuerza de trabajo comunal. En Lolol el $28 \%$ de la fuerza de trabajo eran temporeros que iban a otras comunas y un $21 \%$ equivalente a la fuerza de trabajo lo constituían pensionados. La situación de Teno era parecida: un $24 \%$ corresponde a temporeros y un $22 \%$ a pensionados (11). Más adelante se comentará esta situación al referirnos a las estrategias.

Un último comentario respecto a la migración campo ciudad. Por encuestas realizadas en seis comunas: Curepto, Hualañé, Lolol, Quirihue, Romeral y Teno en un universo de 594 familias en total, se verificó que con leves diferencias no significativas, el promedio del tamaño de las familias rurales era de 5.05 personas por familia. Esto puede apreciarse en los cuadros que siguen. 


\begin{tabular}{|l|l|l|}
\hline \multicolumn{1}{|l|}{ TAMAÑO DE LA FAMILIA RURAL } & & \\
\hline TOTAL ENCUESTA & $\mathbf{5 . 0 5}$ & 4.92 \\
\hline CUREPTO & 5.09 & 5.2 \\
\hline HUALAÑE & 4.92 & 4.95 \\
\hline LOLOL & 5.29 & $\mathbf{5 . 0 9}$ \\
\hline QUIRIHUE & 5.09 & $\mathbf{5 . 0 0}$ \\
\hline ROMERAL & 5.00 & 4.83 \\
\hline TENO & 4.8 & 4.94 \\
\hline & & \\
\hline
\end{tabular}

Valderas y col., Nota al pie de página $\mathrm{N}^{\circ} 12$

TAMAÑO DE LA FAMILIA RURAL Y EN SEDE DE MUNICIPIO

\begin{tabular}{|l|l|l|}
\hline & RURAL & SEDE MUNICIPIO \\
\hline CUREPTO & 5.05 & 5.22 \\
\hline HUALAÑE & 5.24 & 4.53 \\
\hline LOLOL & 5.66 & 4.41 \\
\hline QUIRIHUE & 5.97 & 4.38 \\
\hline ROMERAL & 5.29 & 4.13 \\
\hline TENO & 4.95 & 4.93 \\
\hline
\end{tabular}

Fuente: Valderas y col., Nota al pie de página $\mathrm{N}^{\circ} 12$

Esta constancia en el tamaño de las familias, implica que aun cuando haya varios emigrantes el tamaño tiende a permanecer constante. Esto parece indicar que la organización y medios con que cuenta la familia, en promedio, permite tener ese tipo de estructura soportable de subsistencia, lo cual regula por un lado la población que en el corto plazo tiende a permanecer constante y por otro lado regula en parte la expulsión de los miembros familiares: vale decir la cantidad de emigrantes. Este es un fenómeno de adaptación bastante notable. Por otra parte, al estudiar los grupos familiares con distinto números de emigrantes, durante la existencia de la familia en situación de tener emigrantes, se pudo verificar que cualquiera que fuere el número de emigrantes familiares desde 1 hasta 6 , el tamaño de la familia promedio subsistía(12). Junto con esta constancia se pudo verificar que el promedio de edad de las familias aumentaba con el número de emigrantes desde 28 a 45 años. Esto es una corroboración de que los emigrantes están entre los miembros jóvenes de la familias y efectivamente en la elaboración de la información presentada de estas encuestas ya citadas, en enero de 1997 en el Encuentro de Nacional de Economistas Agrarios (13) se 
estableció, sin lugar a dudas, que las edades de la emigración se encuentran entre los 17 y los 23 años. La emigración en el resto de las edades disminuye en frecuencia, notablemente y se puede verificar que obedece a traslado de matrimonios o esposas o bien por razones de salud. En el caso de los jóvenes, además de las razones de búsqueda de trabajo, estas migraciones coinciden con la salida a estudiar, el período de servicio militar y la formación de una nueva familia como motivo principal; otro incentivo es la conexión previa con algún familiar o amigo que ya haya emigrado.

\section{Cambio parcial de sentido de la movilidad}

Ahora bien, este mismo fenómeno de movilidad puede trasladarse a las capitales de provincia y a los pueblos y aún a localidades pobladas agrícola rurales. Desde hace 20 años se ha constatado que, por efecto de la eliminación del inquilinaje en Chile, muchas familias han ido generando ocupaciones de terrenos contiguos a canales, al salir de los predios donde vivían, o bien se instalaron al lado de los caminos. En otros casos han constituido poblados o villorrios contiguos a la sede de los municipios rurales, (véase Codegua y Romeral, por ejemplo; o por citar una localidad, La Huerta en el camino Curicó -Hualañe). El conocedor del sector rural tiene esa percepción desde hace decenas de años. Por otro lado, el incentivo nacido del traslado de agroindustrias a pueblos relativamente modestos y la necesidad de mano de obra temporal ha incentivado el traslado permanente o temporal de trabajadores o sus familias, creando a la vez un nuevo tipo de transhumancia.

\section{Una nueva realidad comienza a gestarse en Chile}

Ahora se comienza a vivir una nueva forma de movilidad para la gente del campo y la ciudad. Desde el punto de vista comercial es más notoria la ola de condominios en áreas rurales como Pirque, San Bernardo, Isla de Maipo, Calera de Tango; de parcelas de agrado en estos mismos lugares, también en Lampa, Curacaví, en el camino a Santa Juana y Hualqui, en Concepción; en la provincia Valparaíso, donde ya existía un gran mercado asociado a las vacaciones o al veraneo en las playas, y en casi todo el país.

Otro fenómeno menos notorio, pero igualmente importante se está dando por la extensión que puede hacer el campo a la ciudad por la compatibilidad creciente entre tener actividad útil y económica asociada a la tierra y por otro lado tener ocupaciones e intereses en pueblos y ciudades de provincia. Las comunicaciones inalámbricas, los celulares, rastreadores de personas, el cable, caminos pavimentados, el crecimiento de la velocidad promedio en carretera, los fax, la internet, la cercanía de los bancos y servicios, los malls a la salida de las ciudades, etc. hace conveniente reducir los desplazamientos a grandes ciudades y tentar un compromiso entre descanso o tiempo disponible con una fuente de trabajo local o en la periferia de la gran ciudad, incluyendo el ahorro de tiempo que significa no tener que entrar y salir de una gran ciudad que no tiene accesos 
adecuados. Además de la cuestión del tiempo surge el tema de la salud y la comodidad. Si se vive en un pueblo con los servicios relativamente accesibles y confiables se puede trabajar e ir al hogar incluso de a pie. Es más fácil enviar a los hijos al colegio sin tener que sufrir la fatiga y esclavitud o aún el costo de transportar los hijos. Las compras cotidianas pueden también ser más agradables. Ahora por televisión y cable están accesibles aparentemente los productos de la cultura, el arte y el deporte. De esta manera se abre una nueva ola que está asociada, además, a inversiones inmobiliarias y a nuevas comodidades para vivir en ciudades y pueblos de provincia.

Dentro de las nuevas realidades que están emergiendo, se pueden incluir como nueva modalidad del proceso de movilidad poblacional hacia los espacios rurales, al menos tres corrientes:

A. la conservación de los recursos naturales renovables y el equilibrio ecológico B. el dominio y goce del territorio por toda la población, (se habla de descanso, turismo, vacaciones, deporte, gimnasia, actividad física, contemplación, incorporación de la admiración del mundo natural) C. la conveniencia de distribuir más equitativamente los ingresos y la población

\section{Conservación de los recursos y las nuevas tareas u ocupaciones}

Se ha estimado que la erosión causa una pérdida de suelo agrícola de 300 hectáreas al año (14). (Esta cifra es sorprendente dada la gran importancia otorgada al fenómeno por los conservacionistas, ya que esto corresponde a casi a seis cienmilésimos de la tierra agrícola actual, o sea que en 100 años se perderían unas 30.000 hectáreas). El fenómeno parecería sin mucha importancia con relación a la pérdida por urbanización: unas 48.000 hectáreas en 30 años. Los esfuerzos que el país puede hacer en ese lapso para recuperar tierras, detener el desierto y ganarle superficie, avanzar en la utilización de las tierras consideradas improductivas pueden alejar fácilmente el balance negativo que se presenta, a condición de tener una política territorial consistente y permanente. La solución no sería al parecer impedir que la gente viva en el campo pues, si han vivido 3 ó 4 millones de personas en el campo antes, se hace difícil justificar por qué ahora tendríamos que hacer vivir la menor cantidad de gente posible en él. Sin embargo, es posible ocupar el antiguo entorno rural sin erosionar o sin ocupar zonas agrícolas críticas.

Pero el paisaje no está completamente asociado a la agricultura sino al escenario que proporciona la costa, el bosque nativo, las islas, los lagos, las montañas, las nieves, los ventisqueros, los arroyos y vertientes, y el Norte del país que está dominado por las zonas áridas y el desierto.

Pareciera más grave la desertificación y la necesidad de cautelar las reservas forestales, regular los cauces de los ríos, controlando la sedimentación, el embancamiento de los puertos y de ríos y la erosión producida por las lluvias y el 
viento en extensas áreas del territorio, en comparación al tamaño del problema de pérdida del suelo agrícola. Por otra parte, ha comenzado a llegar una corriente de optimismo respecto de la futura disponibilidad de recursos naturales una vez que se superó la crisis energética. Sin embargo, si bien se han debilitado las concepciones apocalípticas, no es menos cierto que es válida la inquietud de dimensionar la herencia que se dejará a las generaciones del futuro. De todos modos las preocupaciones tienen contrapartida en el optimismo proveniente de la invención de innovaciones que han hecho desaparecer muchas nubes maltusianas: la revolución verde, el hallazgo de los nódulos de cobre, el aumento de eficiencias en el uso de materiales y energía, nuevas reservas de minerales y de petróleo, etc.

En Chile, los ríos bajan con velocidad por las pendientes altas entre cordillera y mar, y la aparición de las dunas, advertida por Albert en 1902 (15), como un fenómeno reciente, siendo un fenómeno zooantrópico, originado desde 1850, han afectado suelos que hoy no tienen otro destino que el forestal y en algunos casos han desaparecido, como se puede verificar en Aisén, en la cuenca del río Baker. La erosión es evidente en el secano costero de las VI, VII y VIII regiones. La situación de las extensiones erosionadas en Cautín y Maullín hacen muy preocupante el destino de los suelos chilenos en general. Por lo tanto, el cuidado del territorio adquiere una dimensión distinta a la de otros continentes desde el punto de vista agrícola, pero sobre todo para mejorar y habilitar nuestra residencia en la tierra. Para ello es necesario una ocupación racional del espacio, incluyendo las actuales superficies abandonadas, o vacías, tanto para ejercer soberanía o dominio, como para trabajar concienzudamente por restaurar lo perdido, recuperar y crear hábitats resistentes a la retracción de este patrimonio común. Como este problema ha sido ya internalizado por el chileno común, una política decidida en este sentido tendrá una buena acogida y consecuentemente, tendrá un efecto en los desplazamientos de población.

\section{El goce colectivo del territorio como un derecho que tiende a ejercerse}

El punto que corresponde al goce del territorio es de alta prioridad por la simple razón que la actual población urbana actual, los aproximadamente 13 millones de chilenos de las ciudades deberían disfrutar del uso y aún propiedad de los 740.000 kilómetros cuadrados del Chile continental.

¿Qué significa esto?

Que cada chileno debería tener la posibilidad y la opción real de vivir el paisaje del país en una parte significativa de su tiempo consciente y de una manera grata. Por lo tanto es lícito colocarse en alguno de los casos más generales:

1. Vivir en un lugar rural y trabajar en un pueblo o ciudad durante el año, sin perjuicio de tener períodos cortos destinados a otros puntos.

2. Vivir en un pueblo, o ciudad de servicios o aún capital de provincia y trabajar 
en un área rural que le interese.

3. Alojar y trabajar alternadamente en lugares urbanos y rurales (como ocurre en localidades de la IV Región que proporcionan mano de obra a La Serena).

4. Elegir lugares para reposo de jubilación o enfermedad

5. Tomar vacaciones en distintos lugares ya sea campestres, de montaña o costeros en forma adecuada, evitando una aglomeración forzada del tipo Cartagena en la V Región o de Playa Blanca en la VIII Región.

6. Establecer una complementación más completa entre residencia y posibles alternativas de trabajo

7. Vivir y trabajar en el mundo rural con una fácil complementación urbana, integrada o accesible.

Cuando se plantea así la problemática, ya no sólo es asunto de la agricultura o de la situación de grupos humanos con problemas de marginalidad y pobreza. Integrarse al mundo rural dependerá de la voluntad de estar en contacto con la naturaleza, efectuar acciones relativas al hecho de vivir en ella y a otros factores, por ejemplo la relación residencia-ocupación. Los parques nacionales y las reservas que maneja Corporación Nacional Forestal (CONAF) están creando la posibilidad de hacer realidad una parte de estas alternativas. Conspiran contra el disfrute social, posiblemente, el permitir que muy pocos propietarios tengan el dominio de islas, bosques naturales, cursos de ríos, áreas nevadas, montañas e incluso el espacio actualmente desértico o árido. Sobre esta materia se ha trabajado bastante en el país y en especial en la Escuela de Ciencias Forestales de la Universidad de Chile, en particular en el Departamento de Manejo de Recursos Forestales.

\section{La equidad como complemento a la ocupación y manejo funcional del territorio}

Dentro de las corrientes mencionadas que inciden en la movilidad de la población hacia el sector rural una de ellas se toca con el actual concepto de equidad económica. La equidad ambiental ya está sugerida en el punto seis que se ha expuesto. En efecto, si la preocupación por el territorio está basada en dotar a la toda población del país de una calidad de vida que emerge de un regreso a la naturaleza, del derecho de disfrutar de un bien común que es el entorno físico que nos rodea; este mismo postulado vale para los que viven actualmente en el sector rural o los que deberán vivir allí para cumplir funciones necesarias al resto de la población. Porque el goce de la calidad de vida está asociado, también, al aspecto económico, o sea al ingreso de las familias.

Si se agrega a esto alguna motivación para una colonización racional de lugares deshabitados, se descubrirán tal vez excelentes lugares donde vivir, con la condición de estar relacionados con actividades de servicio y de producción. Ahora, también, entran en la temática los servicios : la red telefónica, la televisión, las escuelas, las postas o la atención primaria de salud, el accesos a bancos y tarjetas de crédito, los supermercados y una serie de servicios que 
cumplen con la función de crear empleos y de hacer la vida grata a los residentes. El sector privado ha hecho avances muy interesantes con los celulares, la televisión o los multicarrier por ejemplo. Desde luego las soluciones habitacionales y la infraestructura que la acompaña, conducen también a la equidad. Ninguna de estas materias son desconocidas por el común de la gente; lo que pasa es que no existe la convicción efectiva de que es necesario ocupar el territorio debido a una conveniencia no sólo considerada como bien común sino que, además sea de conveniencia directa para los diversos grupos de intereses particulares. Si se afirma la idea que despierte interés en los empresarios, trabajadores, sindicatos, habitantes de los diversos lugares, servicios públicos, agencias locales y a la población en general será más factible avanzar en este camino. El buscar una solución intermedia entre lo urbano y lo rural es una perspectiva conveniente y se abre como una nueva ventana para el futuro, pudiendo ser hasta económica. Hace falta un cambio concertado de la visión de vida para consolidar esta nueva corriente hacia el mundo rural. Es probable que en definitiva esto se haga por inmigrantes extranjeros que no han tenido reticencias en el pasado en ocupar los territorios asignados y conquistarlos; sino se hace esto hoy no debería haber quejas por otros, que con esfuerzo vendrán a crear realidades nuevas y a dominarlas.

\section{Instrumentos de análisis para postular cambios y acelerar la comprensión de la situación}

Entretanto, es posible usar algunos instrumentos que desde el punto de vista de los investigadores de la realidad rural ayudan a resolver incógnitas relativas al comportamiento de los actores y a las restricciones que les son inherentes. Paralelamente, se puede seguir prestando atención a fenómenos o características de la vida de las personas o grupos sociales. Algunos de éstos son:

a. Los ciclos de vida de las personas y familias que se han observado ligados a la migración interna. De estos fenómenos tenemos al menos que considerar factores como es el descanso por razones de salud, el matrimonio, la educación, el servicio militar, el inicio de la vida de trabajo, o la relación con parientes y amigos que ya han emigrado.

b. Los circuitos diarios de las personas que hacen soportables los modos de vida, y que limitan o expanden el campo de posibilidades o de elecciones para actividades cotidianas. Estos se refieren preferentemente al uso de estaciones en el tiempo y en el espacio, lo que va creando, por un lado un conjunto de relaciones consecutivas en períodos de tiempo, pero restringidas en el espacio, por una parte, y luego por otra, la limitación de secuencias de actividades distintas, ya sea al azar o rutinarias, debido a la indivisibilidad de las personas que sólo pueden ejecutar una actividad a la vez. La vida compartida como conexión diaria trabajo-alojamiento (switching), con las distintas variantes de alojamiento, ya sea en el campo, 
alojamiento durante la semana en ciudad y fines de semana en el sector rural o viceversa, o trabajo en el campo y residencia en la ciudad son nuevos patrones que influyen en la movilidad y desplazamiento de las personas.

c. Otra línea de pensamiento, que es operativa para vislumbrar la forma de atacar el problema que se propone, es la teoría de la geografía del tiempo de Hägerstrand. En efecto, la sociedad industrial ha elaborado una estructura y capacidad de pronóstico general para asegurar la cooperación entre las personas y las cosas en especial para producir. El sistema es muy potente para producir bienes destinados al consumo privado. Pero no es tan eficiente para suplir otros aspectos de la vida y tiene dificultades para dar a los bienes públicos una participación suficiente. Se ha pasado por alto en alguna medida en el proceso de desarrollo, la necesidad que tiene el ser humano de experimentar coherencia y continuidad en sus actividades y relaciones, tanto en el sentido práctico como en el mental. Tampoco se ha tomado en gran consideración los costos relacionados con ciertas restricciones ambientales de carácter más fundamental que la cantidad existente de materias primas críticas. Se ha establecido una lógica de fragmentación de actividades de toda índole en parcelas cada vez menores. Lo que sucede es que los individuos como pacientes, pupilos, estudiantes se tratan cada vez más en forma separada como si fuesen productos de una fábrica. Por ejemplo, los grupos de edad y las generaciones se orientan hacia agrupamientos separados. Los niños pequeños pierden de vista el mundo adulto en las guarderías infantiles y los ancianos aprenden a morir apropiadamente lejos del resto de la sociedad. Pred hace una explicación muy interesante y completa de este tipo de métodos para el caso sueco. Como un corolario a este aporte Carlstein ofrece una consideración hacia el punto de la localización de las actividades, la asignación de tiempo y espacio para ellas, teniendo en cuenta que se trata de un actor o de actores indivisibles. El conocimiento de la mecánica de las actividades periódicas o diarias, integradas a una manera de vivir con menos stress y con una relación familiar más estable, pueden contribuir a descubrir combinaciones que deriven en un mayor interés colectivo por las residencias rurales con facilidades urbanas que al parecer ya van siendo comunes en el país.

d. La desconcentración de la población.

Este ha sido un tema recurrente abordado por todos los planificadores nacionales, urbanistas y cientistas regionales. En los últimos años la preocupación ha declinado porque se hacen todos los esfuerzos imaginables por invertir en las regiones metropolitanas en obras de descontaminación y descongestión de todo tipo. Al parecer se han considerado dos tipos de realidades: que la migración rural va disminuyendo en importancia porcentual, 
generando las propias urbes su autocrecimiento y por otro que es muy caro o ineficiente, tratar de invertir en la gente o gastar en subsidios para el desarrollo regional y rural. Ambos argumentos disminuyen las probabilidades de desconcentración de la población. Las regiones a su vez hacen esfuerzos por captar nuevas actividades económicas, profesionales y mano de obra con recursos limitados. La expansión de centros superiores de educación en regiones puede aportar un incentivo para la creación de medios sociales y culturales que atraigan habitantes o arraiguen a los que han nacido en las regiones.

\section{La constatación de que se crean estrategias familiares de adaptación para sobrevivir o simplemente para vivir de otro modo en el sector rural}

El examen de diversas situaciones que han sido encontradas permite asegurar que las familias y las personas desarrollan estrategias que les habilitan enfrentar y muchas veces superar las dificultades para conservar el hogar o sobrevivir económicamente.

Por presentar algunos casos, se alude a varios conocidos por el autor: la aplicación de la expectativa de ingreso por parte de los arroceros en Linares, la situación de los campesinos de la zona árida del Limarí, el caso de los agricultores de la zona precordillerana de la VIII región, los temporeros de Lolol, los fabricantes de dulces de Codegua, el agroturismo como actividad más reciente.

Existe no sólo una inercia para cambiarse de residencia, sino que si se dan las condiciones, las familias, aún las que tienen mayor dificultad por las características de su hábitat han encontrado soluciones como la de trasladarse del secano (en la IV Región) para ser temporeros en las zonas de riego, complementando su actividad a veces minera y de ganadería con la agricultura de riego que ha comenzado a desarrollarse. Se ha constatado que la ocupación agrícola ha aumentado en la provincia del Limarí, conjuntamente con la disminución de la ocupación agrícola en el secano. El estudio fue realizado por un equipo mixto del ORSTOM francés y el Centro de Estudios para las Zonas Áridas (Universidad de Chile)

Por otra parte han aparecido nuevas iniciativas como el agroturismo que celebró un Seminario en abril de 1997 en Olmué donde se han compartido las diversas experiencias en el país. Esta modalidad que tiene varias acepciones, dependiendo de su génesis, ha encontrado una receptividad interesante en el país y se pueden describir numerosas combinaciones desde el turismo aventura, pasando por el ecológico para rematar en un disfrute de actividades agrícolas y de entretenimiento en el campo, utilizando alojamientos e infraestructura del campo para pasajeros.

En la Universidad de Concepción Raúl Cerda realizó un interesante trabajo con 
los agricultores de la precordillera de la VIII Región. A partir de una encuesta completa económica y familiar, se demostró que los campesinos de menores recursos agrícolas normalmente utilizan varias opciones para suplir sus necesidades económicas. Entre las más socorridas están la mediería, el trabajo temporal, la venta de su trabajo en el campo y prestación de servicios urbanos. Siempre como en otras comunas ya sea de secano o de zonas áridas, un complemento es la pensión o jubilación.

El último ejemplo que se citará es el de los arroceros cuyas tierras carecen de otras opciones agrícolas. Estos agricultores agregan a sus ingresos la mediería y la práctica agrícola de parte reducida de sus tierras en la introducción de frutales menores, y, también diversas ocupaciones ligadas a la ciudad. Al decir de los expertos éstos agricultores sobre un ingreso promedio de \$ 150.000 mensuales al año pueden decidir permanecer en la actividad; ellos han logrado mantenerse en el mercado por el hecho de haber producido una adaptación de su producción de grano corto a grano largo en un período no superior a los 15 años, dado que existía el "conocimiento" técnico del INIA y el interés de los molineros. Los informes existentes, principalmente de la Estación Quilamapu de Chillán indican que con cambios tecnológicos relativamente simples y de costos muy razonables la actividad arrocera puede subsistir. La esperanza reside justamente en que, con programas de extensión oportunos, se ha pasado en pocos años de un rendimiento promedio de 40 quintales por hectárea 48 quintales por hectárea a nivel nacional en 1996.

Desde 1985, cuando se realizaron las Jornadas Nacionales de Desarrollo Rural en Santiago, no se han hecho intentos tan comprensivos para abordar la realidad del mundo rural. Allí se expusieron los problemas del área desde todos los ángulos posibles, quedando estampadas tanto las implicancias académicas como las económicas y sociales, con diferentes modelos para enfrentar el problema de la pobreza y la degradación del medio natural. Posteriormente se han multiplicado los ensayos y han aparecido estudios más específicos como los citados en este trabajo. La Universidades Austral y de Concepción han acentuado a su dedicación a estos temas, superando la concepción sectorial económica o exclusivamente ambientalista. El Instituto de Investigaciones Agropecuarias y la Corporación Nacional Forestal están contribuyendo en el área productiva, agrícola y silvícola con resultados concretos.

No es aventurado afirmar que se está abriendo un camino interesante para ensayar una prospectiva. A mayor abundamiento, se han popularizado sistemas de información georeferenciales. La mayor parte de las universidades, organizaciones públicas y gobiernos regionales poseen estos instrumentos que permiten obtener imágenes significativas de la distribución de las variables espaciales, avanzando incluso a la representación de los flujos y del dinamismo de diferentes fenómenos. El país, por su parte, ha avanzado tanto en el nivel público como en el privado en sus capacidades de gestión y administración y aflora la esperanza que se pueda distraer una mayor proporción de fondos para 
la equidad. No sólo se hace referencia a la equidad, en el sentido de eliminar la pobreza, sino también a la equidad espacial. Con estos antecedentes es posible imaginar la construcción de una ecuación realista, colectiva para el futuro del mundo rural en Chile.

\section{Notas Bibliográficas}

1. Dickinson, R. City, Region and Regionalism. London, England: Routledge and Kegan Lted, 1947.

2. La Florida pasó desde 18.723 habiotantes en 1960 hasta 328.881 en 32 años, según el Censo XVI de Población, INE, 1992.

3. Maipú experimentó un crecimiento similar al de La Florida en los últimos años, si bien partió desde una base de 51.943 habitantes en 1960 opara llegar en 1992 a los 329.199 según el Censo XVI de Población, INE, 1992.

4. El caso de Puente Alto es muy notable; terminada la expansión explosiva de La Florida, Puente Alto, que era una ciudad de tamaño intermedio, en 10 años más que duplicó su población; subió desde 113.754 en 1982 hasta 254.673 en 1992, según los censos correspondientes yamencionados.

5. La Pintana tenía en 1982, 73.730 habitantes, en 1992 llegó a 169.740 lo que hace un crecimeinto compaable a Puente Alto.

6. Valderas, R., Rustom, A., Valderas, M. Modelo por correspondenciasy programación por metas para la localización urbano-rural de la población de Chile. En: Anales del Tercer Congreso Latinoaericanode Investigación Operativa en Ingeniería de Sistemas, Bosch, M., Weintraub, A (Editores), 1987, tomo I, pp. 37-56.

7. Valderas, R., Contreras, F. y Oliva, J."Antecedentes básicos de la población y superficies comunales de Chile" Documento de Trabajo $N^{\circ} 9$, I.E.R:, Universidad de Chile, Santiago, 1982.

8. Valderas, R. "Característicvas de la población de las comunas chilenas rurales en el periodo 1982-1992" (Documento presentado en el Congreso Agronómico $\mathrm{N}^{\circ} \mathrm{XLVI}$ ), en Simientes (65): 103, $1996 . \quad$ Presentado para publicación en el Departamento de Desarrollo Rural. 14 p., 8 cuadros y 2 figuras.

9. INE. Proyecciones de la ponblación por sexo y edad. Regiones. Fascículo F/CHI, 1987.

10. Valderas, R. y Pérez, A y Royo, A. "Fuerza de trabajo y tendencias migratorias en comunas rurales". en Simiente (60: 105. Sociedad Agronómica de Chile, 1990.

11. Valderas, R. y Pérez, A y Royo, A. op. cit., 1990.

12. Valderas, R. y Pérez, A y Royo, A. "Emigración y cambio de estructura de la familia rural" (Congreso Agronómico $\mathrm{N}^{\circ}$ XLIII, Campus Antumapu, Santiago), en Simiente (62): 105, Sociedad Agronómica de Chile, 1992.

13. Valderas, R., HAmelin, P., Pérez, A. Estrategias familiares y economía de reproducción y mantención en comunas de zonas áridas y de secano. Actas del Encuentro Nacional de Economistas Agrarios, 16-17 de enero 
de 1997, Campus Antumapu, Facultad de Ciencias Agrarias y Forestales, Universidad de Chile, Santiago. En Prensa.

14. Sunkel, Osvaldo. Sustentabilidad ambiental del crecimiento económico chileno. Santiago, Universidad de Chile, 1995.

15. Fuenzalida, V.H. "Suelos",en Geografía Económica de Chile. Ediciones Corporación de Fomento de la Producción, 1967. pp.220-227.

16. Bates, F y Harvey, C. The structure of social systems. New York: Gardner Press Inc., 1975. pp. 263-280.

17. Bates, F y Harvey, C. op.cit.

18. Hägerstrand, T. "El efecto de la organización social y el ambiente sobre el uso del tiempo de los individuos y las familias" en Aspectos sociales de la política y de la planeación regional, A. Kuklinski (editor), F.C.E., México, 1981, pp.63-70.

19. Pred, A. "Urbanización. Problemas de planeación interna e investigación geográfica en Suecia", en Aspectos sociales de la política y de la planeación regional. A. Kuklinski (editor), F.C.E., México, 1981, pp. 288360.

20. Carlstein, T. "¿Sociología regional o espacial?, en Aspectos sociales de la política y de la planeación regiona, A. Kuklinski (editor), F.C.E., México, 1981, PP. 469-4822.

21. Pouget, M.J y otros. Ambiente árido y desarrollo sustentable: la provincia de Limarí, ed. Ceza-Orstom, Santiago, Chile, 1996.

22. Pérez, A. Agroturismo. Aspectos socioeconómicos y de la identidad social", en Anales de la Universidad de Chile, VI serie, N 1997.

23. Cerda, R. Aspectos socioecoómicos de la agricultura tradicional de la precordillera andina de la Octava Región. Actas del Encuentro Nacional de Economistas Agrarios, 16-17 de enero de 1997, Campus Antumapu, Facultad de Ciencias Agrarias y Forestales, Universidad de Chile, Santiago. EN prensa.

24. Valderas, R. Economía de u cultivo tradicional: el arroz. Trabajo in extenso presentado en el XLVII Congreso Agronómico, Campus San Joaquín, Universidad Católica, 29-30 de noviembre, Santiago, 1996.

25. Departamento de Desarrollo Rural. "Jornadas Nacionales de Desarrollo Rural", en Boletín Serie de Desarrollo Rural 20, Universidad de Chile. Facultad de Ciencias Agrarias y Forestales, Santiago, Chile, 1985. 